\title{
Massively Parallel Anisotropic Mesh Adaptation Applied to Earth Land Elevation
}

\author{
Digonnet H. ${ }^{*},{ }^{\dagger}$ Aissa N. ${ }^{\dagger}$, Douteau L. ${ }^{\dagger}$ and Silva L. ${ }^{\dagger}$ \\ † École Centrale de Nantes \\ High Performance Computing Institute \\ 1 rue de la Noë, 44300 Nantes, France \\ e-mail: hugues.digonnet@ec-nantes.fr, web page: https://ici.ec-nantes.fr/
}

\begin{abstract}
In this paper, we will present the work and results obtained during a "Grands Challenges" session on a French supercomputer, Irène Joliot-Curie, as well as an application to Earth land elevation using the SRTM data [1] as input. The goal of such a project was to increase the scalability of our mesher [2] at a larger computing scale (several dozens of thousands of cores) and so validate the good scalability of the application. A second objective concerned the possibility of graphically exploit such huge results.

Both isotropic and anisotropic benchmarks have been done. For the isotropic part, the goal was to build the largest possible mesh, with more than 1000 billion elements. Building such a mesh needed the use of, at least, 65536 cores and up to 200 TB of memory. An anisotropic application case has also been done, to well capture the Earth's surface elevation at full scale. Using a high resolution SRTM image, we have first generated 16-billion adapted faces representing the Earth surface. Then, the anisotropic mesh adaptation procedure, including computation of a signed distance to the set of faces, of an error estimator, of the metric construction and topological mesh adaptation, has been run using 1384 cores.
\end{abstract}
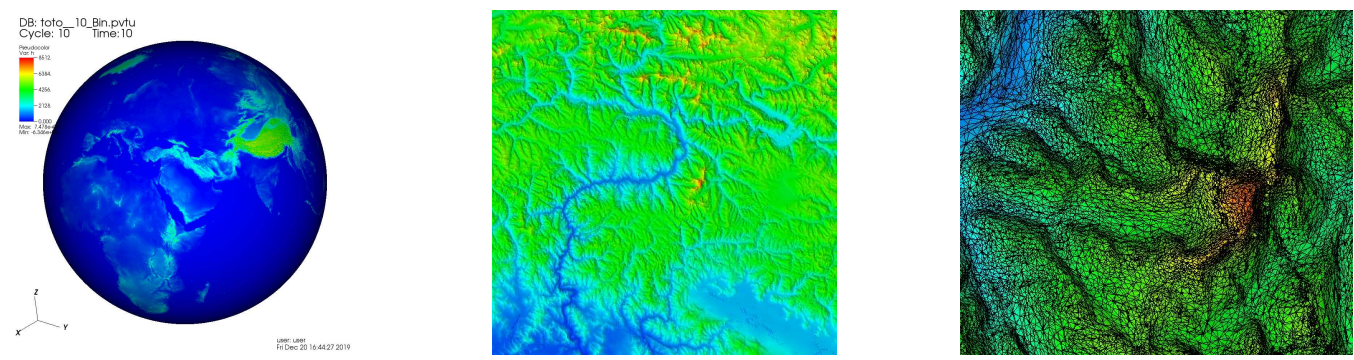

Figure 1: Three levels of zoom representation of the zero isovolume of the LevelSet function, isovolume colored by the sea-level elevation on Earth. It is related to the final 3d (volume) anisotropic adapted mesh and enables to well represent the Earth's surface given by a highly defined SRTM input. It has about 40billion elements and has been run on more than 8192 cores. This mesh keeps details of less than 100 meters of size, with an element size that varies between less than $1 \mathrm{~m}$ and $50 \mathrm{~km}$, inside a global 4096 billion $\mathrm{km}^{3}$ cube

\section{REFERENCES}

[1] Jarvis A., H.I. Reuter, A. Nelson, E. Guevara, Hole-filled seamless SRTM data V4, International Centre for Tropical Agriculture (CIAT), available from http://srtm.csi.cgiar.org. (2008)

[2] Digonnet, H. and al. Massively parallel anisotropic mesh adaptation. Int. J. High Perf. Comp. App., Vol. 33(1) pp. 3-24, (2017). 